\title{
Adaptive Modulation with Best User Selection over Non-Identical Nakagami Fading Channels
}

\author{
Anlei Rao and Mohamed-Slim Alouini \\ King Abdullah University of Science and Technology (KAUST) \\ Thuwal, Mekkah Province, Saudi Arabia \\ E-mail:\{anlei.rao;mohamed.alouini\}@kaust.edu.sa
}

\begin{abstract}
In this paper, we analyze the performance of adaptive modulation with single-cell multiuser scheduling over independent but not identical distributed (i.n.i.d.) Nakagami fading channels. Closed-form expressions are derived for the average channel capacity, spectral efficiency, and bit-error-rate (BER) for both constant-power variable-rate and variable-power variable-rate uncoded $M$-ary quadrature amplitude modulation (M-QAM) schemes. We also study the impact of time delay on the average BER of adaptive M-QAM. Selected numerical results show that the multiuser diversity brings a considerably better performance even over i.n.i.d. fading environments.
\end{abstract}

\section{INTRODUCTION}

To meet the growing demand of services for wireless communications with relatively scarce spectrum, adaptive modulation [1] has been proposed for the last two decades as an effective method of increasing the link spectral efficiency. When the channel side information can be estimated and this estimation sent back to the transmitter, the transmit rate and power can be adapted relative to the channel characteristics to achieve better performance. In [2], the channel capacity with channel side information was derived for both constantpower variable-rate (CPVR) and variable-power variable-rate (VPVR) transmission. In [3], a VPVR M-ary quadrature amplitude modulation (M-QAM) scheme was proposed for data transmission over fading channels. This paper showed that there is a constant power gap between the channel capacity and the spectral efficiency for this scheme, and this gap is a function of the maintained bit-error-rate (BER). In [4], variablerate adaptation for M-QAM with constant-power allocation has been analyzed over the Nakagami fading channels.

In a single-cell multiuser environment, multiuser diversity [5] takes advantage of the variability in fading channels to improve the overall spectral efficiency. For instance, consider that a reasonably large number of users experiencing independent time-varying fading conditions are actively transmitting or receiving data in a given cell. By granting the channel access only to the user with the best transmission quality, we can achieve a diversity gain so that an increase in the channel capacity and spectral efficiency can be reached. Different multiuser scheduling algorithms were proposed in [5], [6] to choose the best user and reduce the feedback load. In this paper, we simply select the user with the largest instantaneous singal-to-noise ratio (SNR) to perform data transmission. With the best user selected, we analyze the channel capacity, average spectral efficiency and average BER for both CPVR and VPVR schemes with M-QAM over an independent but not necessarily identical distributed (i.n.i.d.) Nakagami fading channels.

The operation of the CPVR and VPVR schemes needs accurate channel estimation at the receiver, a reliable feedback path between the estimator and transmitter, and a negligible time delay. So an efficient error control scheme is therefore needed to insure the transmitter's access of the channel side information. In this paper, we assume perfect channel estimation and a reliable feedback, and we also quantify the effect of time delay on the proposed system under consideration. In addition, it has been shown in [4] that the time delay affects the average BER due to the variability of the channel conditions. As such, the goal is to keep the time delay under a threshold to limit the impact on the BER.

The rest of this paper is organized as follows. In section II, the multiuser model is introduced. Next, section III first reviews the channel capacity and spectral efficiency of both CPVR and VPVR schemes, and then gives closed-form expressions for them with best user selection over i.n.i.d. Nakagami fading channels. The performance of the adaptive M-QAM is also analyzed in this section. In section IV, we consider the impact of time delay on the average BER. Finally, some numerical results are shown in section $\mathrm{V}$ and conclusions are drawn in the last section.

\section{SySTEM MODEL}

Consider a single-cell multiuser environment with $N$ users uniformly distributed in a cell of radius $R$ centered around a base station. In this case, the average SNR $\bar{\gamma}$ for each user is assumed to be log-normal distributed with a shadow standard deviation of $\theta \mathrm{dB}$ and an average SNR $\bar{\gamma}_{R}$ at distance $R$, and a mean value following an exponentially decreasing path loss model with an exponent $\zeta$. In this case, the probability density function (PDF) for the average SNR $\bar{\gamma}$ is given by [7], [8]:

$f_{\bar{\gamma}}(\bar{\gamma})=\frac{2}{c} \exp \left\{\frac{2 \theta^{2}-2 c\left(\bar{\gamma}-\bar{\gamma}_{R}\right)}{c^{2}}\right\} Q\left(\frac{2 \theta^{2}-c\left(\bar{\gamma}-\bar{\gamma}_{R}\right)}{c \cdot \theta}\right)$,

where $c=10 \zeta \log _{10}(e)$ is the exponential path loss parameter, $Q(\cdot)$ is the Gaussian $Q$-function defined as the tail probability of the standard normal distribution, i.e. $Q(x)=$ $\frac{1}{\sqrt{2 \pi}} \int_{x}^{\infty} \exp \left(-\frac{u^{2}}{2}\right) d u$.

At the beginning of each time slot, the user with the largest instantaneous SNR is selected to perform data transmission 
in this time slot. Let $\gamma_{i}$ denote the instantaneous SNR for the $i$-th user and user $b$ as the selected best user such that $b=\arg \max _{i \in \mathcal{R}}\left\{\gamma_{i}\right\}$, where $\mathcal{R}=\{1,2, \cdots, N\}$. We can then write $\gamma_{b}=\max \left\{\gamma_{1}, \gamma_{2}, \cdots, \gamma_{N}\right\}$.

Now suppose that the channels between the users and the base station are slowly-varying flat-fading such that the channel condition changes at a rate much slower than the symbol data rate. In this case, the channel remains roughly constant over hundreds of symbols. For Nakagami fading channels, the SNR of each user $\gamma_{i}$ is Gamma distributed with the parameters $\left(\bar{\gamma}_{i}, m_{i}\right)$ and the PDF of $\gamma_{i}$ is thus given by [9]

$$
f_{\gamma_{i}}(\gamma)=\left(\frac{m_{i}}{\bar{\gamma}_{i}}\right)^{m_{i}} \frac{\gamma^{m_{i}-1}}{\Gamma\left(m_{i}\right)} \exp \left(-\frac{m_{i}}{\bar{\gamma}_{i}} \gamma\right), \quad \gamma \geq 0,
$$

where $\Gamma(\cdot)$ is the Gamma function.

It is easy to show the cumulative distribution function (CDF) of the instantaneous SNR of the best user can be written as

$$
F_{\gamma_{b}}(\gamma)=\prod_{i=1}^{N} F_{\gamma_{i}}(\gamma)=\prod_{i=1}^{N}\left(1-\frac{\Gamma\left(m_{i}, \frac{m_{i}}{\bar{\gamma}_{i}} \gamma\right)}{\Gamma\left(m_{i}\right)}\right), \gamma \geq 0 \text {, }
$$

where $\Gamma(\cdot, \cdot)$ is the upper incomplete gamma function. Restricting $m_{i}$ 's to integers and using the relation $\Gamma(n, x)=$ $\Gamma(n) \mathrm{e}^{-x} \sum_{i=0}^{n-1} \frac{x^{i}}{i !}$, the CDF of $\gamma_{b}$ can then be expressed for integer $m_{i}$ 's as

$$
F_{\gamma_{b}}(\gamma)=\sum_{S} \sum_{L}(-1)^{K} C_{S, L} \gamma^{l_{S}} \cdot \mathrm{e}^{-E_{S} \gamma}, \gamma \geq 0,
$$

where $S=\left\{s_{1}, s_{2}, \cdots, s_{K}\right\}$ is a subset of $\mathcal{R}, \sum_{S}$ is the sum over all possible $S, \sum_{L}$ is the sum defined by

$$
\sum_{L}=\sum_{l_{s_{1}}=0}^{m_{s_{1}}-1} \sum_{l_{s_{2}}=0}^{m_{s_{2}}-1} \cdots \sum_{l_{s_{K}}=0}^{m_{s_{K}}-1} .
$$

In (3), $l_{S}, E_{S}$ and $C_{S, L}$ are given by $l_{S}=l_{s_{1}}+l_{s_{2}}+\cdots+l_{s_{K}}$, $E_{S}=\frac{m_{s_{1}}}{\bar{\gamma}_{s_{1}}}+\frac{m_{s_{2}}}{\bar{\gamma}_{s_{2}}}+\cdots+\frac{m_{s_{K}}}{\bar{\gamma}_{s_{K}}}$, and

$$
C_{S, L}=\prod_{i=1}^{K} \frac{1}{l_{s_{i}} !}\left(\frac{m_{s_{i}}}{\bar{\gamma}_{s_{i}}}\right)^{l_{s_{i}}} .
$$

When $S=\phi$ is an empty set, we have $l_{S}=0, E_{S}=0$ and $C_{S, L}=1$. By simply differentiating (3) over $\gamma$, the PDF of $\gamma_{b}$ can then be written as

$f_{\gamma_{b}}(\gamma)=\sum_{S} \sum_{L}(-1)^{K} C_{S, L}\left(l_{S}-E_{S} \gamma\right) \gamma^{l_{S}-1} \mathrm{e}^{-E_{S} \gamma}, \gamma \geq 0$.

\section{Performance Analysis}

Assuming perfect channel estimation and reliable feedback, the time-varying channel capacity of a fading channel $\langle C\rangle$ $[\mathrm{Bits} / \mathrm{Sec}]$ with variable power allocation and rate adaptation is given ny [2]

$$
<C>=\max _{P(\gamma)}\left\{\int_{0}^{\infty} W \log _{2}\left(1+\gamma \frac{P(\gamma)}{\bar{P}}\right) f_{\gamma}(\gamma) d \gamma\right\}
$$

where $W[\mathrm{~Hz}]$ is the channel bandwidth, $f_{\gamma}(\gamma)$ is the distribution of the received SNR, $\bar{P}$ is the average transmit power, and $P(\gamma)$ is the instantaneous transmit power chosen relative to $\gamma$, which is subject to the power constraint

$$
\int_{0}^{\infty} P(\gamma) f_{\gamma}(\gamma) d \gamma \leq \bar{P}
$$

If we choose uncoded M-QAM for the adaptive modulation scheme of choice, the BER with variable power allocation can be then well approximated by $\operatorname{BER}(M, \gamma) \simeq$ $0.2 \exp \left(-\frac{3 \gamma}{2(M-1)} \frac{P(\gamma)}{\bar{P}}\right)$ [3]. To maintain a fixed BER of $\mathrm{BER}_{0}$, the constellation size $M$ is adjusted as

$$
M=1+\frac{\gamma}{K_{0}} \frac{P(\gamma)}{\bar{P}}
$$

where $K_{0}=-\frac{2}{3} \ln \left(5 \mathrm{BER}_{0}\right)$.

\section{A. Constant-Power Variable-Rate Adaptation}

1) Channel Capacity: With a constant transmit power $P(\gamma)=\bar{P}$, the channel capacity $\langle C\rangle_{\mathrm{cpvr}}$ is given as

$$
<C>_{\text {cpvr }}=W \int_{0}^{\infty} \log _{2}(1+\gamma) f_{\gamma}(\gamma) d \gamma .
$$

Substituting (4) in (8) yields the channel capacity per unit bandwidth over i.n.i.d. Nakagami fading channels with the best user selection

$$
\begin{aligned}
<\frac{C}{W}>_{\mathrm{cpvr}} & =\log _{2}(\mathrm{e}) \sum_{S} \sum_{L}(-1)^{K} C_{S, L} \\
& \left(l_{S} \mathcal{I}_{l_{S}}\left(E_{S}\right)-E_{S} \mathcal{I}_{l_{S}+1}\left(E_{S}\right)\right),
\end{aligned}
$$

where $\mathcal{I}_{n}(\mu)$ is defined in [10, (32)], and its closed-form evaluation for a positive integer $n$ is also derived in [10, (78)].

2) Spectral Efficiency: With the constant power scheme and $M$ chosen in (7), the spectral efficiency for continuous-rate MQAM can be approximated by [4]

$$
<\frac{R}{W}>_{\mathrm{cpvr}}=\int_{0}^{\infty} \log \left(1+\frac{\gamma}{K_{0}}\right) f_{\gamma}(\gamma) d \gamma,
$$

Substituting (4) in (10), the above spectral efficiency per unit bandwidth can be written as

$$
\begin{aligned}
<\frac{R}{W}>_{\mathrm{cpvr}} & =\log _{2}(\mathrm{e}) \sum_{S} \sum_{L}(-1)^{K} C_{S, L} K_{0}^{l_{S}} \\
& \left(l_{S} \mathcal{I}_{l_{S}}\left(E_{S} K_{0}\right)-E_{S} K_{0} \mathcal{I}_{l_{S}+1}\left(E_{S} K_{0}\right)\right) .
\end{aligned}
$$

3) Constellation Restriction: For practical use, the constellation size $M$ is often restricted to $2^{n}$ for a positive integer $n$. We divide the whole SNR range into $T+1$ fading regions, and assign $M_{n}$ as the constellation size to the $\mathrm{n}$-th region. When the estimated SNR falls in the $n$-th region, $M_{n}$ is chosen for M-QAM. With the constellation size $M_{n}$ and the boundary SNRs $\gamma_{n}$ chosen as the same as in [4, (30)], we have the spectral efficiency as the sum of the data rates $\left(\log _{2}\left(M_{n}\right)\right)$ associated with each region multiplied by the probability of the instantaneous SNR $\gamma$ falling in that region

$$
<\frac{R}{W}>_{\mathrm{cpdr}}=\sum_{n=1}^{T} r_{n} p_{n},
$$




$$
\overline{\mathrm{BER}}_{n}=\sum_{S} \sum_{L}(-1)^{K} \frac{C_{S, L}}{5 \beta_{S_{n}}^{l_{S}}}\left(l_{S} \Gamma\left(l_{S}, \beta_{S_{n}} \gamma_{n}, \beta_{S_{n}} \gamma_{n+1}\right)-\frac{E_{S}}{\beta_{S_{n}}} \Gamma\left(l_{S}+1, \beta_{S_{n}} \gamma_{n}, \beta_{S_{n}} \gamma_{n+1}\right)\right)
$$

where $r_{n}=\log _{2}\left(M_{n}\right)=n, p_{n}=\int_{\gamma_{n}}^{\gamma_{n+1}} f_{\gamma_{b}}(\gamma) d \gamma=$ $F_{\gamma_{b}}\left(\gamma_{n+1}\right)-F_{\gamma_{b}}\left(\gamma_{n}\right)$, which can be easily computed with (2).

For continuous-rate M-QAM, it always operates at the target $\mathrm{BER}_{0}$. When the constellation size is restricted to some integer values, however, the discrete M-QAM operates at an average $<$ BER $>_{\text {cpdr }}$ lower than $\mathrm{BER}_{0}$. Similar to the calculation of the spectral efficiency, the average BER with constellation restriction is the ratio of the average number of bits in error over the total average number of transmitted bits [4]

$$
<\mathrm{BER}>_{\mathrm{cpdr}}=\frac{\sum_{n=1}^{T} r_{n} \overline{\mathrm{BER}}_{n}}{\sum_{n=1}^{T} r_{n} p_{n}},
$$

where $\overline{\mathrm{BER}}_{n}$ is calculated by

$$
\overline{\operatorname{BER}}_{n}=\int_{\gamma_{n}}^{\gamma_{n+1}} \operatorname{BER}\left(M_{n}, \gamma\right) f_{\gamma_{b}}(\gamma) d \gamma
$$

Substituting $\operatorname{BER}\left(M_{n}, \gamma\right)=\frac{1}{5}\left(5 \mathrm{BER}_{0}\right)^{\frac{\gamma}{\gamma_{n}}}$ into (14), we have the $\overline{\mathrm{BER}}_{n}$ shown on the top of this page as (15), where $\beta_{S_{n}}=$ $E_{S}+1.5 K_{0} / \gamma_{n}$, and $\Gamma(n, a, b)=\Gamma(n, a)-\Gamma(n, b)$.

\section{B. Variable-Power Variable-Rate Adaptation}

1) Channel Capacity: By optimizing the channel capacity (5) with the power constraint (6), we have the channel capacity with optimal power allocation as [2]

$$
<C>_{\mathrm{vpvr}}=W \int_{\gamma_{0}}^{\infty} \log _{2}\left(\frac{\gamma}{\gamma_{0}}\right) f_{\gamma}(\gamma) d \gamma,
$$

where $\gamma_{0}$ is the optimal cutoff SNR below which data transmission is suspended and can be determined by solving $\int_{\gamma_{0}}^{+\infty}\left(\frac{1}{\gamma_{0}}-\frac{1}{\gamma}\right) f_{\gamma}(\gamma) d \gamma=1$. It can be rewritten in terms of the $\mathrm{CDF}$ of $\gamma_{b}$ as

$$
\begin{aligned}
& \frac{1-F_{\gamma_{b}}\left(\gamma_{0}\right)}{\gamma_{0}}=1+\sum_{S} \sum_{L}(-1)^{K} C_{S, L} \\
& E_{S}^{1-l_{S}}\left(l_{S} \Gamma\left(l_{S}-1, E_{S} \gamma_{0}\right)-\Gamma\left(l_{S}, E_{S} \gamma_{0}\right)\right)
\end{aligned}
$$

When $l_{s}=0$ or 1 , we have $\Gamma\left(0, E_{S} \gamma_{0}\right)=E_{1}\left(E_{S} \gamma_{0}\right)$, where $E_{1}(\cdot)$ is the exponential integral of the first order [11], and $F_{\gamma_{b}}\left(\gamma_{0}\right)$ can be easily evaluated using (2).

By substituting (4) in (16), the channel capacity per unit bandwidth with optimal power allocation can be written as

$$
\begin{aligned}
<\frac{C}{W}>_{\mathrm{vpvr}}= & \log _{2}(\mathrm{e}) \sum_{S} \sum_{L}(-1)^{K} C_{S, L} \gamma_{0}^{l_{S}} \\
& \left(l_{S} \mathcal{J}_{l_{S}}\left(E_{S} \gamma_{0}\right)-E_{S} \gamma_{0} \mathcal{J}_{l_{S}+1}\left(E_{S} \gamma_{0}\right)\right),
\end{aligned}
$$

where the definition of $\mathcal{J}_{n}(\mu)$ is given in [10, (14)], and its closed-form evaluation for a positive integer $n$ is also derived in $[10,(64)]$.
2) Spectral Efficiency: With the optimal power allocation and $M$ chosen in (7), the spectral efficiency is given by [3]

$$
<\frac{R}{W}>_{\mathrm{vpvr}}=\int_{\gamma_{K}}^{\infty} \log _{2}\left(\frac{\gamma}{\gamma_{K}}\right) f_{\gamma}(\gamma) d \gamma,
$$

where $\gamma_{K}$ is the cutoff fade depth under which no power is allocated to transmit data, which is determined by $K_{0} \int_{\gamma_{K}}^{+\infty}\left(\frac{1}{\gamma_{K}}-\frac{1}{\gamma}\right) f_{\gamma}(\gamma) d \gamma=1$

The spectral efficiency with optimal power allocation over i.n.i.d. Nakagami fading channels can be derived by simply replacing $\gamma_{0}$ in (18) with $\gamma_{K}$

$$
\begin{aligned}
<\frac{R}{W}>_{\mathrm{vpvr}}=\log _{2}(\mathrm{e}) \sum_{S} \sum_{L}(-1)^{K} C_{S, L} \gamma_{K}^{l_{S}} \\
\left(l_{S} \mathcal{J}_{l_{S}}\left(E_{S} \gamma_{K}\right)-E_{S} \gamma_{K} \mathcal{J}_{l_{S}+1}\left(E_{S} \gamma_{K}\right)\right) .
\end{aligned}
$$

3) Constellation Restriction: Similar to the constellation restriction in the CPVR scheme, we also divide the whole SNR range into $T+1$ fading regions, but restrict our constellation size as $M_{0}=0, M_{1}=2$, and $M_{n}=2^{2(n-1)}, n=2, \cdots, T$. The boundary SNR $\gamma_{n}$ is determined by $\gamma_{n}=\gamma^{*} M_{n}$ with a parameter $\gamma^{*}>0$ which satisfies the power constraint (21) if we adopt the same power allocation scheme as in [3]

$$
\sum_{n=1}^{T}\left(M_{n}-1\right) K_{0} \int_{\gamma^{*} M_{n}}^{\gamma^{*} M_{n+1}} \frac{f_{\gamma_{b}}(\gamma)}{\gamma} d \gamma=1 .
$$

The spectral efficiency can also be calculate by (12) with $p_{n}=F_{\gamma_{b}}\left(\gamma^{*} M_{n+1}\right)-F_{\gamma_{b}}\left(\gamma^{*} M_{n}\right)$ and $r_{1}=1, r_{n}=2(n-$ $1), n=2,3, \cdots, T$. And the BER will always operate at $\mathrm{BER}_{0}$, i.e. $<\mathrm{BER}>_{\mathrm{vpdr}}=\mathrm{BER}_{0}$.

\section{EFFECTS OF TIME DELAY}

Recall that the choice of the constellation size $M$ and power allocation $P(\gamma)$ are based on a channel estimation at time $t$, while the data are sent over the channel at time $t+\tau$, where $\tau$ is the time delay for the channel estimation and feedback. If the time delay $\tau$ degrades the BER significantly, then the adaptive technique will not work. Assuming perfect channel estimation and reliable feedback, we analyze in this section the impact of time delay on BER with the best user selection in an i.n.i.d. Nakagami fading environment.

We denote $\rho$ as the correlation factor between the SNR $\gamma$ at time $t$ and the SNR $\gamma_{\tau}$ at time $t+\tau . \rho$ can be expressed in terms of time delay $\tau$, the mobile speed $v$, and the wavelength of the carrier frequency $\lambda_{c}$ by $\rho=J_{0}^{2}\left(2 \pi f_{D} \tau\right)$ [12], where $J_{0}(\cdot)$ is the zero-order Bessel function of the first kind, and $f_{D}=v / \lambda_{c}$ is the maximum Doppler frequency shift. With $\rho$, the conditional PDF of $\gamma_{\tau}$ over $\gamma_{b}$ is given by [9]

$$
\begin{aligned}
& f_{\gamma_{\tau} \mid \gamma_{b}}\left(\gamma_{\tau} \mid \gamma\right)=\frac{m_{b}}{(1-\rho) \bar{\gamma}_{b}}\left(\frac{\gamma_{\tau}}{\rho \gamma}\right)^{\frac{m_{b}-1}{2}} \times \\
& \exp \left(-\frac{m_{b}}{\bar{\gamma}_{b}} \frac{\rho \gamma+\gamma_{\tau}}{1-\rho}\right) I_{m_{b}-1}\left(\frac{2 m_{b} \sqrt{\rho \gamma \gamma_{\tau}}}{(1-\rho) \bar{\gamma}_{b}}\right),
\end{aligned}
$$

where $m_{b}$ and $\bar{\gamma}_{b}$ are the Nakagami parameter and average SNR for the best user $b$, respectively, and $I_{n}(\cdot)$ is the modified Bessel function of the first kind. 


$$
<\mathrm{BER}_{\tau}>_{\mathrm{cpvr}}=\frac{1}{5} \sum_{S} \sum_{L}(-1)^{K} C_{S, L}\left(l_{S} \mathcal{F}_{m_{b}}^{0, \infty}\left(\alpha, \beta, l_{S}-1, E_{S}\right)-E_{S} \mathcal{F}_{m_{b}}^{0, \infty}\left(\alpha, \beta, l_{S}, E_{S}\right)\right)
$$

$$
\overline{\mathrm{BER}}_{n}=\frac{1}{5}\left(\frac{\gamma_{n}}{\alpha+\gamma_{n}}\right)^{m_{b}} \sum_{S} \sum_{L}(-1)^{K} \frac{C_{S, L}}{\alpha_{S_{n}}^{l_{S}}}\left(l_{S} \Gamma\left(l_{S}, \alpha_{S_{n}} \gamma_{n}, \alpha_{S_{n}} \gamma_{n+1}\right)-\frac{E_{S}}{\alpha_{S_{n}}} \Gamma\left(l_{S}+1, \alpha_{S_{n}} \gamma_{n}, \alpha_{S_{n}} \gamma_{n+1}\right)\right)
$$

\section{A. Constant Power Scheme}

With the constant power allocation, the instantaneous BER is given by $\operatorname{BER}\left(\gamma, \gamma_{\tau}\right)=\frac{1}{5}\left(5 \mathrm{BER}_{0}\right)^{\frac{\gamma \gamma}{\gamma}}$. Averaging it over (22) yields the conditional average BER on $\gamma$ as

$$
\operatorname{BER}(\gamma)=\frac{1}{5}\left(\frac{\gamma}{\gamma+\alpha}\right)^{m_{b}} \exp \left(-\frac{\beta \gamma}{\gamma+\alpha}\right)
$$

where $\alpha=\frac{3(1-\rho) K_{0} \bar{\gamma}_{b}}{2 m_{b}}$ and $\beta=\frac{3}{2} K_{0} \rho$. Averaging $\operatorname{BER}(\gamma)$ over the PDF of $\gamma_{b}$ given in (4) yields the average BER for CPVR scheme accounting for the impact of the time delay as (23) shown on the top of this page, where $\mathcal{F}_{m}(\alpha, \beta, a, b)$ is defined as

$\mathcal{F}_{m}^{x, y}(\alpha, \beta, a, b)=\int_{x}^{y}\left(\frac{\gamma}{\gamma+\alpha}\right)^{m} \exp \left(-\frac{\beta \gamma}{\gamma+\alpha}\right) \gamma^{a} \mathrm{e}^{-b \gamma} d \gamma$.

Considering the restriction of the constellation, the instantaneous BER yields $\operatorname{BER}\left(M_{n}, \gamma_{\tau}\right)=\frac{1}{5}\left(5 \mathrm{BER}_{0}\right)^{\frac{\gamma_{\tau}}{\gamma_{n}}}$. Averaging this expression over the conditional PDF (22) yields the conditional average BER on $\gamma$ for the discrete rate as

$$
\operatorname{BER}_{n}(\gamma)=\frac{1}{5}\left(\frac{\gamma_{n}}{\gamma_{n}+\alpha}\right)^{m_{b}} \exp \left(-\frac{\beta \gamma}{\gamma_{n}+\alpha}\right),
$$

The average BER is calculated by (13) with the $\overline{\mathrm{BER}}_{n}$ calculated by (14), the result of which is shown as (24) on the top of this page, where $\alpha_{S_{n}}=E_{S}+\frac{\beta}{\alpha+\gamma_{n}}$ with $\alpha$ and $\beta$ given as above.

\section{B. Variable Power Scheme}

For the variable power scheme, the power allocation $P(\gamma) / \bar{P}$ is chosen so that it always operates at the target BER. Taking time delay into consideration, the instantaneous BER, for both the continuous rate and discrete rate, yields $\operatorname{BER}\left(\gamma, \gamma_{\tau}\right)=\frac{1}{5}\left(5 \mathrm{BER}_{0}\right)^{\frac{\gamma \tau}{\gamma}}$.

For the continuous-rate VPVR scheme, the data is transmitted only when the instantaneous SNR is above the cutoff SNR, or $\gamma>\gamma_{K}$. So the average BER for continuous-rate VPVR scheme with time delay effect is given by

$$
\begin{array}{r}
<\mathrm{BER}_{\tau}>_{\mathrm{vpvr}}=\frac{1}{5} \sum_{S} \sum_{L}(-1)^{K} C_{S, L}\left(l_{S} \mathcal{F}_{m_{b}}^{\gamma_{K}, \infty} \times\right. \\
\left.\left(\alpha, \beta, l_{S}-1, E_{S}\right)-E_{S} \mathcal{F}_{m_{b}}^{\gamma_{K}, \infty}\left(\alpha, \beta, l_{S}, E_{S}\right)\right) .
\end{array}
$$

With the constellation restriction, the average BER is calculated by (13), where $p_{n}=F_{\gamma_{b}}\left(\gamma^{*} M_{n+1}\right)-F_{\gamma_{b}}\left(\gamma^{*} M_{n}\right)$, $r_{1}=1, r_{n}=2(n-1), n=2,3, \cdots, T$, and $\overline{\mathrm{BER}}_{n}$ is given in this case by

$$
\begin{aligned}
& \overline{\mathrm{BER}}_{n}=\frac{1}{5} \sum_{S} \sum_{L}(-1)^{K} C_{S, L}\left(l_{S} \mathcal{F}_{m_{b}}^{\gamma_{n}, \gamma_{n+1} \times}\right. \\
&\left.\left(\alpha, \beta, l_{S}-1, E_{S}\right)-E_{S} \mathcal{F}_{m_{b}}^{\gamma_{n}, \gamma_{n+1}}\left(\alpha, \beta, l_{S}, E_{S}\right)\right) .
\end{aligned}
$$

\section{Numerical Results}

In the following figures, we consider a single-cell multiuser environments with a shadow standard deviation of $\theta=3 \mathrm{~dB}$ and a path-loss exponent $\zeta=3$. The average SNR $\bar{\gamma}_{i}$ 's for each user are supposed to be i.n.i.d. following the distribution given in (1) with a cell-range SNR $\bar{\gamma}_{R}$. In our results, the Nakagami parameter for each user is fixed as $m=3$.

In Fig. 1, we plot the channel capacity and spectral efficiency for different number of users for both the CPVR and VPVR schemes. We can see in the figure that the channel capacity and spectral efficiency for CPVR and VPVR schemes are almost the same. With the constellation restriction, the spectral efficiency for CPVR scheme degrades considerably, while for the VPVR scheme, the effect of constellation restriction is offset by the continuous power allocation. Note that all the users are distributed in a single cell with the cellrange SNR $\bar{\gamma}_{R}=0 \mathrm{~dB}$. When the number of users increases, we maintain the $\bar{\gamma}_{R}$ unchanged, so that the cell radius remains the same. We can see from the figure that both the channel capacity and spectral efficiency are marginally decreasing.

In Fig. 2 and Fig. 3, the channel capacity and spectral efficiency for different cell-range SNR $\bar{\gamma}_{R}$ are shown for the CPVR and VPVR schemes, respectively. In Fig. 2, we see a saturation of the spectral efficiency at large SNR area for the discrete-rate CPVR scheme. For the large SNR range, the current constellation size is not enough for transmission with a higher efficiency, and larger constellation size is frequently used to achieve better performance. While in Fig. 3, the power allocation for discrete-rate VPVR scheme will be highly suboptimal for large SNR. In this circumstances, the power is far from being fully utilized and no $\gamma^{*}$ is found as the solution for (21), so that no results for large SNR are shown in the figure. In order to utilize the power fully, larger constellation size is also needed to achieve a better performance.

Finally in Fig. 4, the impact of time delay on BER is studied. From the figure we can see that, if the normalized delay is maintained under a value of $10^{-2}$, the impact of delay is tolerable and the systems will be able to operate satisfactorily. Beyond that, the time delay will degrade the BER significantly. Comparing the curves of $\mathrm{BER}_{0}=10^{-3}$ and $\mathrm{BER}_{0}=10^{-6}$, we can find that the smaller the $\mathrm{BER}_{0}$ we want to maintain, the more sensitive the BER is to the time delay.

\section{CONCLUSION}

In this paper, we analyze the CPVR and VPVR schemes over the i.n.i.d. Nakagami fading channels when the best user is chosen in a single-cell environment. Close-form expressions have been derived for average channel capacity, spectral efficiency, and BER with uncoded M-QAM. Numerical results show that by selecting the best user, we can still improve the performance considerably through the diversity gain. In 
addition, we also studied the impact of time delay on BER, and numerical results show that when the normalized time delay is below $10^{-2}$, the impact is tolerable. However, the BER degrades considerably when the normalized time delay exceeds $10^{-2}$.

\section{REFERENCES}

[1] S. Sampei, S. Komaki, and N. Morinaga, "Adaptive modulation/tdma scheme for personal multimedia communication systems," in Proc. of Global Telecommunications Conference (GLOBECOM'94), pp. 989993, San Francisco, CA, USA, November, 1994.

[2] A. J. Goldsmith and P. P. Varaiya, "Capacity of fading channels with channel side information," IEEE Transactions on Information Theory, vol. 43, no. 6, pp. 1986-1992, June, 1997.

[3] A. J. Goldsmith and S. G. Chua, "Variable-rate variable-power MQAM for fading channels," IEEE Transactions on Communications, vol. 45, no. 10 , pp. 1218-1230, October, 1997.

[4] M.-S. Alouini and A. J. Goldsmith, "Adaptive modulation over Nakagami fading channels," Wireless Personal Communications, vol. 13, no. 1, pp. 119-143, January, 2000.

[5] R. Knopp and P. A. Humblet, "Information capacity and power control in single-cell multiuser communications," in Proc. of IEEE International Conference on Communications (ICC'95), pp. 331-335, Seattle, WA USA, June, 1995.

[6] D. Gesbert and M.-S. Alouini, "How much feedback is multi-user diversity really worth?" in Proc. of IEEE International Conference on Communications (ICC'04), pp. 234-239, Paris, France, June, 2004.

[7] L. Yang, M. Kang, and M.-S. Alouini, "On the capacity-fairness tradeoff in multiuser diversity systems," IEEE Transactions on Vehicular Technology, vol. 56, no. 4, pp. 1901-1907, April, 2007.

[8] M. D. Yacoub, Foundations of Mobile Radio Engineering. CRC Press, Inc. Boca Raton, FL, USA, 1993.

[9] M. Nakagami, "The m-distribution-A general formula of intensity distribution of rapid fading," Statistical Method in Radio Propagation, pp. 3-36, Pergamon Press, Oxford, England, 1960.

[10] M.-S. Alouini and A. J. Goldsmith, "Capacity of Rayleigh fading channels under different adaptive transmission and diversity-combining techniques," IEEE Transactions on Vehicular Technology, vol. 48, pp. 1165-1181, July, 1999.

[11] I. S. Gradshteyn and I. M. Ryzhik, Table of Integrals, Series, and Products, San Diego, CA: Academic Press, fifth ed., 1994.

[12] W. C. Jakes, Microwave Mobile Communications, New York: Wiley, 1974.

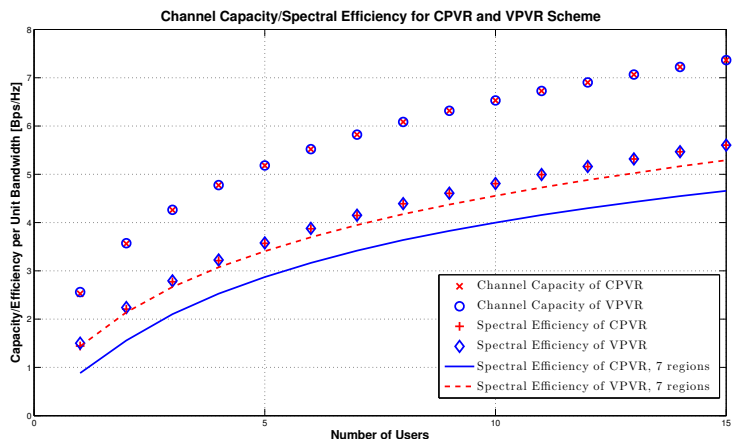

Figure 1. Channel capacity and spectral efficiency for different number of users $\left(\bar{\gamma}_{R}=0 \mathrm{~dB}\right.$ and $\left.\mathrm{BER}_{0}=10^{-6}\right)$.

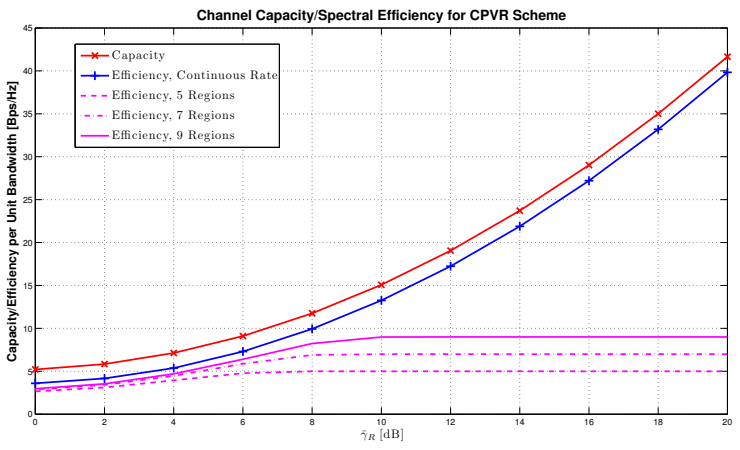

Figure 2. Channel capacity and spectral efficiency for different values of cell-range SNR $\bar{\gamma}_{R}$ for the CPVR scheme $\left(N=5\right.$ and $\left.\mathrm{BER}_{0}=10^{-6}\right)$.

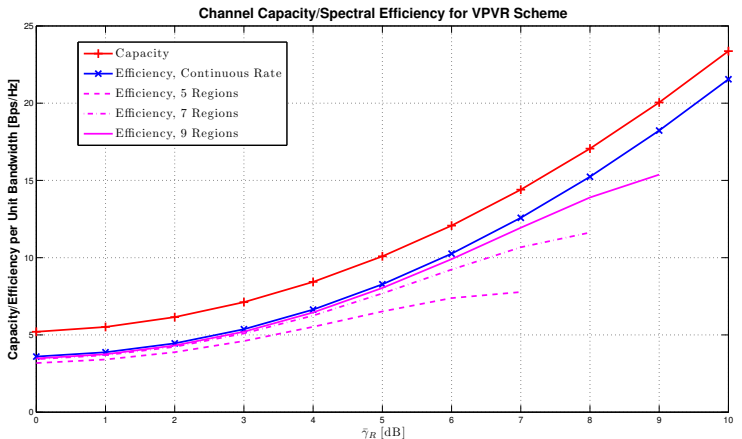

Figure 3. Channel capacity and spectral efficiency for different values of cell-range SNR $\bar{\gamma}_{R}$ for the VPVR scheme $\left(N=5\right.$ and $\left.\mathrm{BER}_{0}=10^{-6}\right)$.

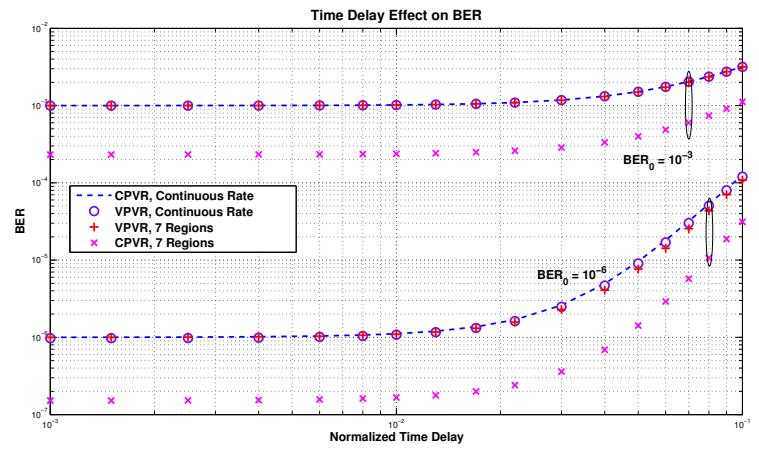

Figure 4. Time delay effect on the BER for different values of normalized time delay $\left(\bar{\gamma}_{R}=0 \mathrm{~dB}\right.$ and $\left.N=5\right)$. 